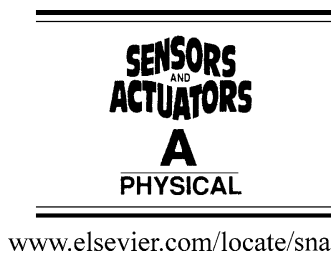

\title{
Microactuators based on ion implanted dielectric electroactive polymer (EAP) membranes
}

\author{
Philippe Dubois ${ }^{\mathrm{a}, \mathrm{d}, *}$, Samuel Rosset ${ }^{\mathrm{a}}$, Sander Koster ${ }^{\mathrm{b}}$, Johann Stauffer ${ }^{\mathrm{c}}$, \\ Serguei Mikhaïlov ${ }^{c}$, Massoud Dadras ${ }^{d}$, Nico-F. de Rooij ${ }^{d}$, Herbert Shea ${ }^{a}$ \\ a Microsystems for Space Technologies Laboratory, Ecole Polytechnique Fédérale de Lausanne, Lausanne, Switzerland \\ ${ }^{\mathrm{b}}$ University of Groningen, Groningen, The Netherlands \\ ${ }^{\mathrm{c}}$ Centre d'analyse par faisceau ionique (CAFI), Le Locle, Switzerland \\ ${ }^{\mathrm{d}}$ Institute of Microtechnology (IMT), University of Neuchâtel, Switzerland
}

Received 10 November 2005; accepted 25 November 2005

Available online 19 January 2006

\begin{abstract}
We report on the first successfully microfabricated and tested ion implanted dielectric electroactive polymer (DEAP) actuators. Dielectric EAP (DEAP) actuators combine exceptionally high energy-density with large amplitude displacements [S. Ashley, Artificial muscles, Sci. Am. 289 (2003) 52-59; R. Pelrine, R. Kornbluh, J. Joseph, R. Heydt, Q. Pei, S. Chiba, High field deformation of elastomeric dielectrics for actuators, Mater. Sci. Eng. C 11 (2000) 89-100]. Scaling DEAPs down to the millimeter and micron scale requires patterning compliant electrodes on such a scale on the surfaces of the polymer. We used ion implantation to make the surfaces of the polymer locally conducting. Implanting the compliant electrodes solves the problem of microfabricating patterned electrodes whose elasticity is close to that of the insulating elastomer, thus avoiding the deposition of metal electrodes on the polymer which leads to significant stiffening of the membrane [A. Pimpin, Y. Suzuki, N. Kasagi, Micro electrostrictive actuator with metal compliant electrodes for flow control applications, in: MEMS 04, Maastricht, The Netherlands, 25-29 January, 2004, pp. 478-481]. Several techniques based on ion implantation for chip level and wafer level fabrication are presented. Ion implanted DEAP membranes were both simulated (FEM) and characterized. We report measurements on an actuator consisting of a 30- $\mu \mathrm{m}-\mathrm{thick}$ ion implanted PDMS membrane bonded to a silicon chip into which a cavity had been etched. We measured $110 \mu \mathrm{m}$ vertical displacements for a $0.72 \mathrm{~mm}{ }^{2}$ membrane, achieving for the first time the same percent displacement in microscopic DEAPs as in macroscopic devices. These observations show that ion implantation allows the patterning of electrodes on PDMS membranes with negligible increase in stiffness.
\end{abstract}

(c) 2005 Elsevier B.V. All rights reserved.

Keywords: Ion implantation; Dielectric electroactive polymer; EAP; DEAP; Dielectric elastomer actuator; DEA

\section{Introduction}

Microactuators based on stiff materials such as silicon generally have a very limited out of plane displacements. Using elastic materials such as elastomers instead allows much larger displacements. This fact is exploited in macro-sized dielectric elastomer actuators (DEA) [1,2]. However, the several attempts conducted worldwide to scale down these devices to the mm or $\mu \mathrm{m}$ range have encountered major difficulties, mostly related to the micropatterning of sufficiently compliant electrodes [2-4]. We present here a novel method to microfabricate compliant elec-

\footnotetext{
* Corresponding author. Tel.: +41 32720 5266; fax: +41 327205754 .

E-mail address: philippe.dubois@unine.ch (P. Dubois).
}

trodes by using implantation of metal ions into polymers to make the elastomer locally conductive without significantly increasing its stiffness. By implanting only in specific micrometer-sized areas, one can create and individually address many independent large displacement DEAs on a single chip, allowing for complex actuation schemes.

The working principle of a dielectric EAP is based on the compression of a dielectric elastomer membrane by the electrostatic pressure of compliant soft electrodes [1,2]. The compression of the elastomer results in an elongation of the elastomer without a change in volume (Fig. 1) [5].

Depending on the boundary conditions (what the membrane is attached to) and on the material properties of the membrane, the membrane either buckles, bends or elongates in-plane (Fig. 2) [2]. A DEA consists of a dielectric EAP (DEAP) mem- 

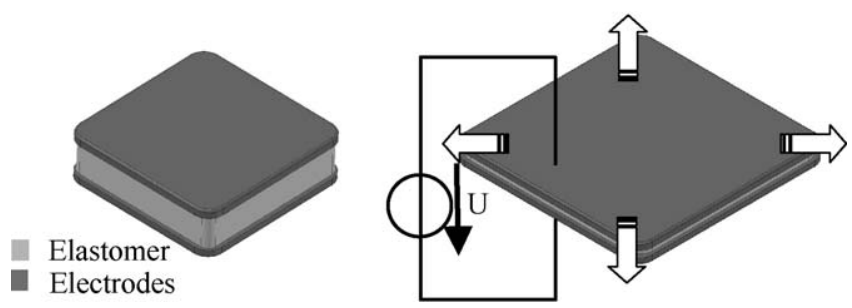

Fig. 1. Dielectric EAP (DEAP) principle [1]. When a voltage $U$ is applied to the electrodes, the electrostatic pressure squeezes the elastomer dielectric (right side). The volume of the dielectric being quasi constant, the whole structure stretches. Maximum strains of over $100 \%$ have been reported [1,2].

brane fixed to a rigid body at well-defined locations [1]. The motion of the DEAP membrane can then be translated into the actuation of other structures such as robotic arms, grippers and orientating devices or used directly to interact with liquid, gases or even the human body [1].

Macro size DEAPs are based on unpatterned compliant electrodes connected by wires $[1,2,5]$. To reduce the size of the device and to increase functionality and integration, it would be extremely desirable to pattern the electrodes in order to address many DEAPs on one single membrane [6]. Localized metallic ion implantation on the surface of an elastomer will enable independently addressing several DEAP actuators on one single membrane (Fig. 3).

The field of low energy ion implantation into polymers is still not mature. A few publications report on resistivity reduction by ion implantation into polymers [7-13]. These studies concern rigid polymers such as PET or PTFE. Only metallic ion implantation is reported to induce large reduction of polymer surface resistivity $[7,12,13]$. Wu et al. compared surface resistivity for different metallic ions implanted into PET at relatively low energies $(<50 \mathrm{keV})$ and doses as large as $2 \times 10^{17}$ atoms $/ \mathrm{cm}^{2}$ [7]. Such combination of large doses and low energy requires

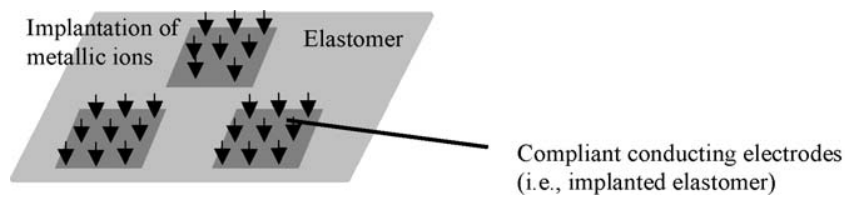

Fig. 3. Implantation of metallic ions into elastomeric membranes to create localized compliant electrodes and EAPs.

the use of particular implantation techniques such as plasma immersion ion implantation (PIII).

To our best knowledge, DEA micro-actuators based on ion implantation have never been reported in the literature.

\section{Background and motivation}

Vertical displacements of approximately $10 \%$ of the membrane diameter have been reported for macro scale diaphragmtype DEAPs [2]. Prior to this work, the best micro fabricated DEAP membranes showed displacements limited to about $1.5 \%$ of the diameter using spring type metallic electrodes to minimize electrode stiffness [3]. This lower value for the micro-scale devices was attributed by Pimpin et al. to the stiffness of the metallic compliant electrodes [3]. The Young's modulus of soft polymers is typically four orders of magnitude lower than most metals. This explains the low performance of micro-DEA using metal electrodes: metal electrodes 10 -nm-thick will have stiffness comparable to a $100-\mu \mathrm{m}$-thick elastomer film. In contrast, we expect ion implanted polymer electrodes to have a similar stiffness to the unimplanted polymer. This is the key to efficient device miniaturization.

In order to create micro DEAs, we bonded ion implanted membranes onto micromachined silicon substrates. The silicon structure supports the membrane at specific locations and thus defines the boundary conditions. The actuators reported

(a) Buckling mode
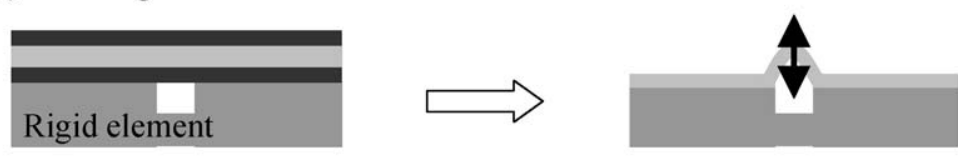

(b) Bending mode
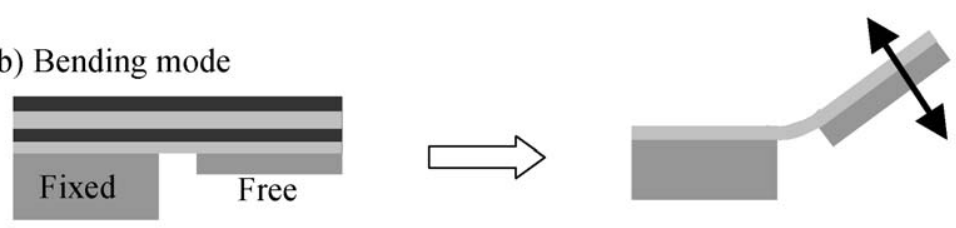

(c) In plane mode
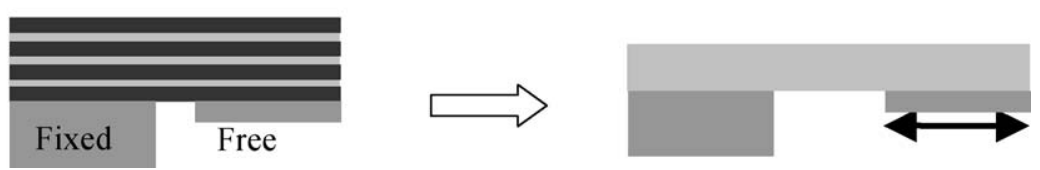

Fig. 2. Three different actuation modes of a DEA [2]. (a) When electrodes are created on both surfaces of the elastomer over a cavity, the membrane tries to elongate and eventually buckles when actuated. (b) In case of a buried electrode, the membrane bends when actuated. (c) A thick DEAP membrane consisting of a stack of many layers can be used for in-plane actuation. 

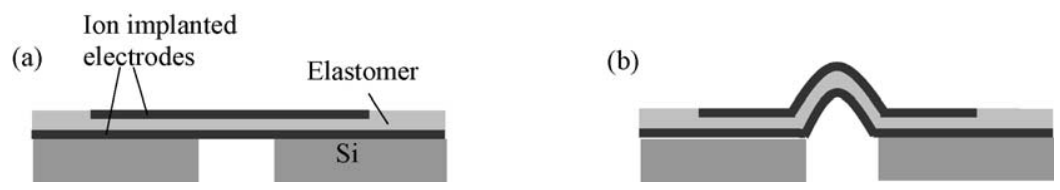

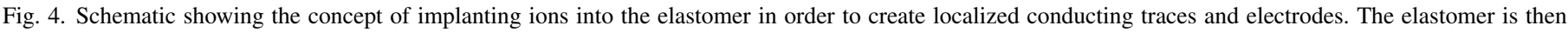

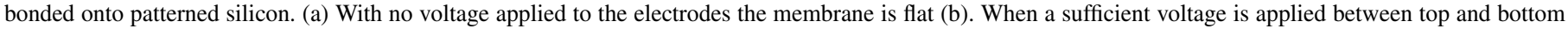
electrodes, the membrane buckles on top of the cavity etched into the silicon.

in this article were designed to create a buckling of the ion implanted dielectric electroactive polymer membrane (DEAP) when actuated. The DEAP membrane is bonded to the silicon chip and covers an opening (etched through the chip) to define the boundary conditions, as shown schematically in Fig. 4a. When actuated, the membrane buckles either upward or downward (Fig. 4b). The direction of displacement (buckling), i.e. upward or downward, depends on asymmetries in the structure and boundary conditions as well as the presence of external forces such as gravity or pressure. The symmetry can be broken by burying one of the implanted conducting layers inside the PDMS. Such geometry favours one specific direction of displacement. The mode of buckling that enables the largest displacement is the mode that has only one maximum, i.e. the first mode.

\section{Design considerations and simulations}

Design parameters of the DEAP include the orifice geometry, electrode geometry (size and location), operation voltage, and film thickness. By choosing a suitable polymer one has some control over the Young's modulus $Y$, electrical breakdown field $E_{\text {bd }}$ and dielectric permittivity $\varepsilon$ [2]. While one could seek to maximize the applied force, one often seeks to maximize the displacement, which requires maximizing the compression of the elastomer.

For a free standing DEAP membrane, the elastomer membrane compression depends on its dielectric permittivity $\varepsilon$, its thickness, its Young's modulus $Y$ and the actuation voltage $U$ [2]. The main practical factor limiting the electrostatic compression of a DEAP membrane is the electrical breakdown of the polymer, which occurs at the critical electric field $E_{\mathrm{bd}}$. The higher the actuation voltage, the thinner the DEAP membrane becomes, which increases the applied electric field, and hence enhances risk of electrical breakdown.

In the absence of electrical breakdown, for non-pre-stretched membranes, a localized collapse of the membrane is reported between 30 and $40 \%$ compression of the membrane [6]. This is similar to the pull-in effect observed for parallel plate electrostatic actuator. For a linear material (stiffness independent of strain) the collapse point corresponds to vertical strain $s_{Z}$ of $33 \%$ [6]. In experiments it is observed that the thinning is localized due to defects. This localized thinning of the membrane then often results in the destruction of the DEAP membrane by electrical breakdown as the thinning can increase the electric field above $E_{\mathrm{bd}}$ [6]. Some elastomers with low $E_{\mathrm{bd}}$ and high Young's modulus $Y$ could reach electrical breakdown before the mechanical collapse point.
We focused on modelling DEAP diaphragm-type actuators, i.e. a membrane on top of an orifice, as in Fig. 4. The actuation voltage for maximum displacement depends on the boundary conditions, geometry and initial internal stress in the membrane. In order to model the displacement of diaphragm DEAP membrane we introduced the Young's modulus value measured on macroscopic samples $\left(Y_{\mathrm{PDMS}}\right.$ SmoothOn $=0.17 \mathrm{MPa}$, see macroscopic material testing section) into 3D FEM simulations of displacement (ANSYS). For square orifices, the membrane buckles according to its first mode (Fig. 5).

Our FEM simulations of diaphragm membranes showed a strong dependence on parameters including $Y$, pre-strain, $\varepsilon$, and boundary conditions. In our case, namely an ion implanted PDMS membrane glued onto a silicon chip, some of theses parameters are not well known. For example, the validity of using the macroscopic Young's modulus for thin $(10-30 \mu \mathrm{m})$ PDMS membranes is not proven. Comparison with other measurement methods would have to be realized to confirm these values. The fabrication process can induce pre-strain of the membrane and the permittivity coefficient used may not be accurate. We used a geometrically non-linear model and deliberately ignored any possible stiffening of the membrane due to ion implantation. Therefore these simulations give essentially a qualitative appreciation of the shape of the deflected membrane.

Simulations showed a displacement of $110 \mu \mathrm{m}$ of square 30$\mu \mathrm{m}$-thick DEAP membrane measuring $850 \mu \mathrm{m} \times 850 \mu \mathrm{m}$ for an actuation voltage of $400 \mathrm{~V}$ (Fig. 5).

\section{Macroscopic material testing}

We measured the Young's modulus in compression with a standard macroscopic apparatus for material testing (Schenk). The test samples (SmoothOn, length $=14.4 \mathrm{~mm}$, diameter $=25.2 \mathrm{~mm}$; Sylgard 186 , length $=14.6 \mathrm{~mm}$, diameter $=20.7 \mathrm{~mm}$ ) were compressed by two parallel metallic plates lubricated with silicone grease. We tested two different PDMS elastomer, Sylgard 186 (Dow Corning) and Dragon Skin (SmoothOn). In the literature, Sylgard 186 is reported to have a Young's modulus $Y=0.7 \mathrm{MPa}$ [2].

The stress was measured for true strains of up to $-90 \%$, but we defined the Young's modulus as the slope of the compression curve for $s_{\mathrm{Z}}$ varying from -10 to $-20 \%$ [14]. We observed that Sylgard 186 from DowCorning (fitted for $s_{\mathrm{Z}}=-10$ to $-20 \%$, $Y=1.02 \mathrm{MPa}$ ) is much stiffer than DragonSkin from SmoothOn (fitted for $\mathrm{s}_{\mathrm{Z}}=-10$ to $-20 \%, Y=0.17 \mathrm{MPa}$, Fig. 6). The presence of bubbles in the test samples, especially for the Sylgard 186 that is highly viscous, adds some uncertainties to these measurements. 


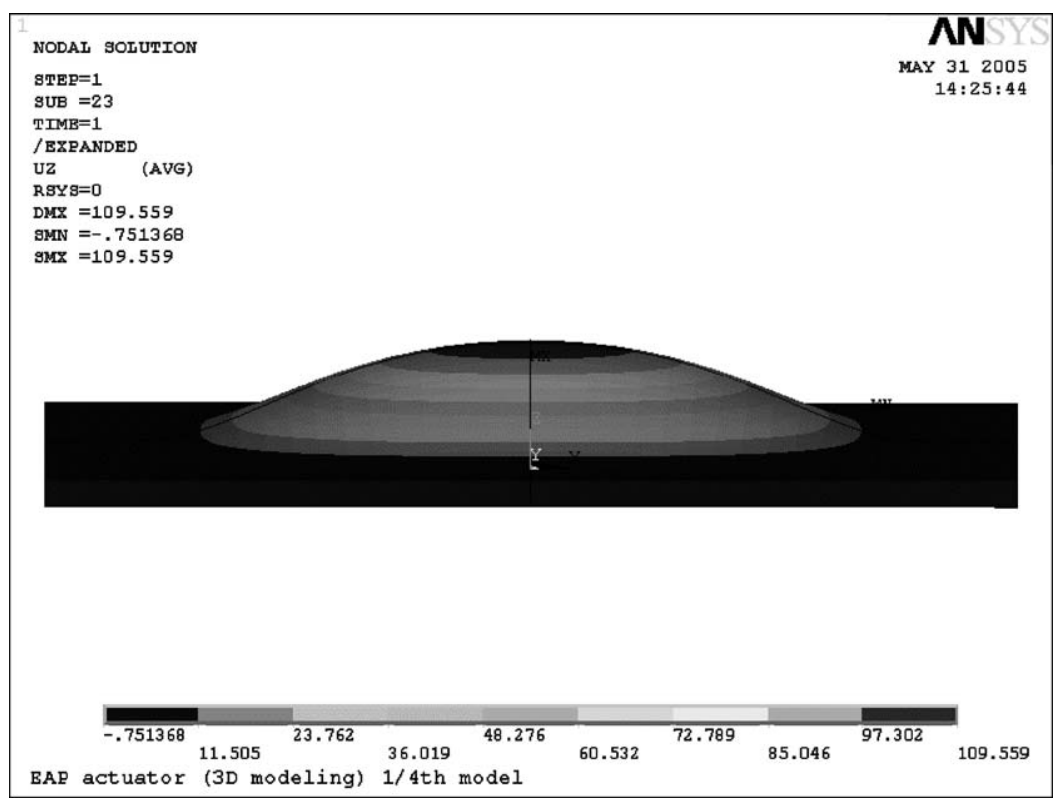

Fig. 5. Geometrically non-linear multi-physics displacement FEM simulation of a 30- $\mu \mathrm{m}$-thick EAP membrane measuring $850 \mu \mathrm{m} \times 850 \mu \mathrm{m}$ actuated with $400 \mathrm{~V}$. The displacement of the central point is $110 \mu \mathrm{m}$. Conditions: $Y_{\text {SmoothOn }}=0.17 \mathrm{MPa}, \varepsilon=3$, no pre-strain.

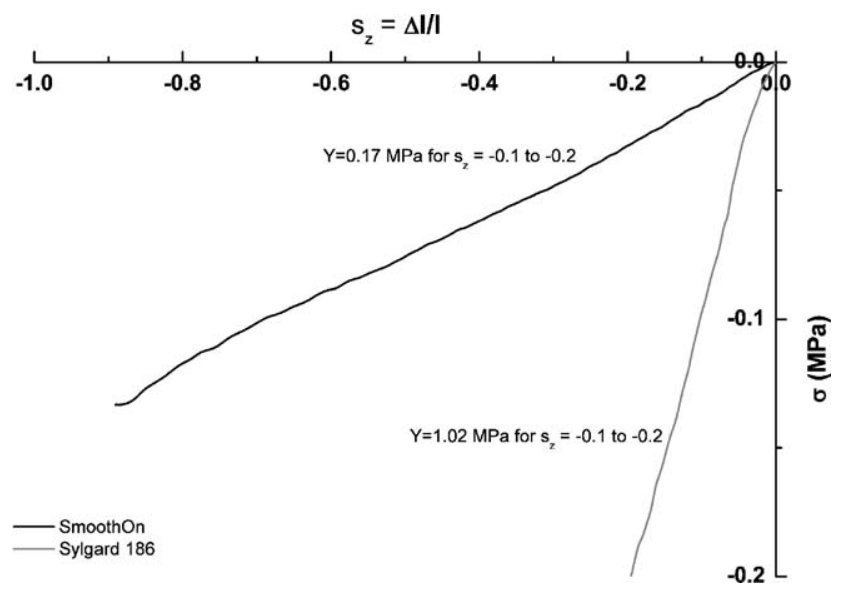

Fig. 6. Macroscopic compression tests of two different elastomers (PDMS), Sylgard 186 (Dow Corning) and Dragon Skin (SmoothOn). True stress is plotted vs. true strain.

\section{Device fabrication}

For the actuator reported here, the dielectric consists of a PDMS membrane bonded to a silicon chip with rectangular and square through holes ranging in area from 0.72 to $3 \mathrm{~mm}^{2}$. $\mathrm{KOH}$ wet etching was used to process the larger holes and deep reactive ion etching (DRIE) for the smaller ones. Ion implantation was carried out on both sides of the PDMS membranes. We fabricated the devices with a chip-scale (rather than wafer scale) process mostly because the ion implanter we used can only implant over an area of $1 \mathrm{~cm}^{2}$. A wafer scale process is described at the end of this section.

The 30- $\mu \mathrm{m}$-thick PDMS membranes were made by spinning soft PDMS (SmoothOn, Dragon Skin) onto a transfer silicon wafer covered with a thin homogenous water-soluble acrylic sacrificial layer [15] (Fig. 7a). This PDMS was diluted at a 1:1 $(\mathrm{v} / \mathrm{v})$ ratio into isooctane and spun at $1000 \mathrm{rpm}$ during $30 \mathrm{~s}$. The Ti ion implantation was carried out in a filtered cathodic vacuum arc implanter (FCVA) at the CAFI (Le Locle, Switzerland) with an acceleration voltage of $2.5 \mathrm{keV}$. We used such a low energy in order to minimize the damage of the PDMS surface and to implant ions to a depth of about 10-nm. This laboratory implantor does not provide a direct indication of the implanted dose. We estimated the dose based both on the resistivity of the implanted PDMS and on simulations of our structure using the SRIM software (developed by J.F. Ziegler). For implantation of Ag in PET at a dose of $2 \times 10^{17}$ atoms $/ \mathrm{cm}^{2}$, Wu et al. report resistivities of about $0.01 \Omega \mathrm{m}$. We obtained surface resistivities below $100 \mathrm{k} \Omega / \square$ (below $0.05 \Omega \mathrm{m}$ based on implantation depth simulations), so we estimate our implanted doses at above $1 \times 10^{17}$ atoms $/ \mathrm{cm}^{2}$.

The implanted side of the PDMS film was then bonded or glued (implanted side down) onto the pre-processed silicon chips (Fig. 7b). Bonding by surface preparation using an $\mathrm{O}_{2}$ plasma for 6-18 s resulted in inconsistent adhesion in the implanted areas. Therefore, in the more recent chips the plasma bonding process was replaced by gluing using as an adhesive the identical PDMS used for the membrane. Once the silicon chips were fixed to the PDMS membrane, the PDMS membranes were cut manually around the silicon chips and detached from their silicon support by dissolving the acrylic sacrificial layer (PAA) in water. Finally, the top side of the PDMS was ion implanted using a polyimide mask to define the top electrode geometry (Fig. 7c). For testing, the polyimide was detached (Fig. 7d).

Two different sizes of chip and orifices were fabricated. A larger device with a rectangular orifice of $1.5 \mathrm{~mm} \times 2.0 \mathrm{~mm}$ was fabricated using plasma $\mathrm{O}_{2}$ for bonding the membrane (Fig. 8). A smaller device was made by gluing (rather than $\mathrm{O}_{2}$ plasma bonding) ion implanted membranes on smaller chips having an orifice of $850 \mu \mathrm{m} \times 850 \mu \mathrm{m}$ (Fig. 9). 


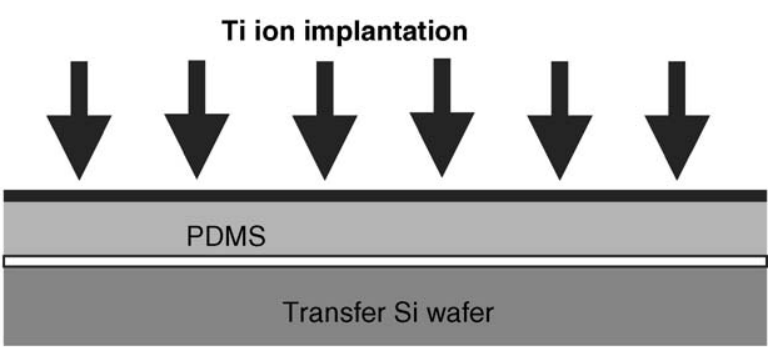

(a)

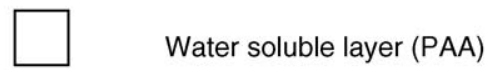

(b)

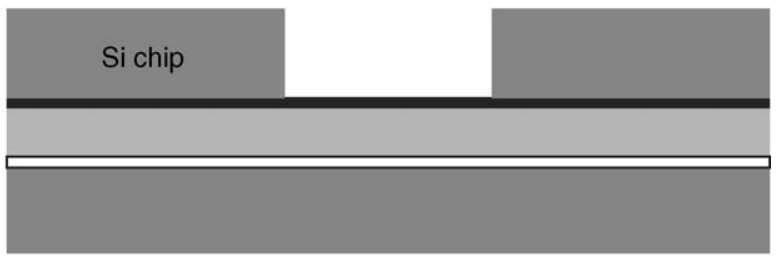

(c)
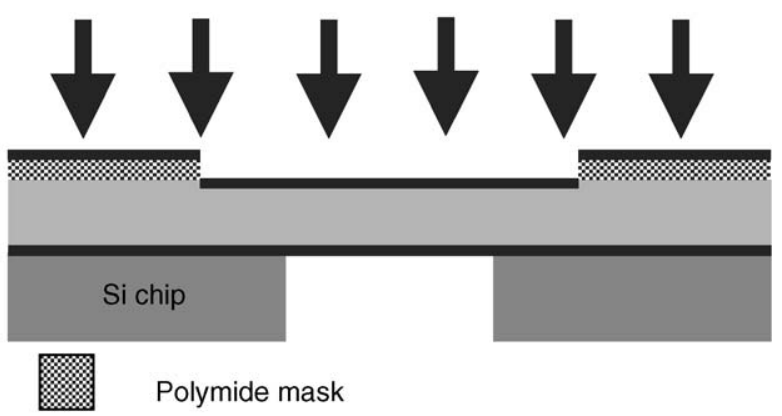

(d)

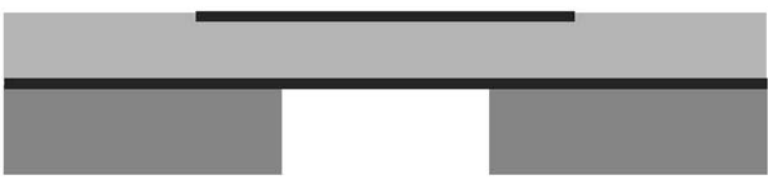

Fig. 7. Chip-scale process flow used for the microfabrication of symmetrical ion implanted PDMS diaphragm DEAP actuators. (a) Implantation of PDMS film on transfer wafer. (b) Bonding to patterned Si wafer. (c) Removal of the transfer wafer, patterning PDMS with polyimide, implanting second side of PDMS. (d) Striping of the polyimide mask.

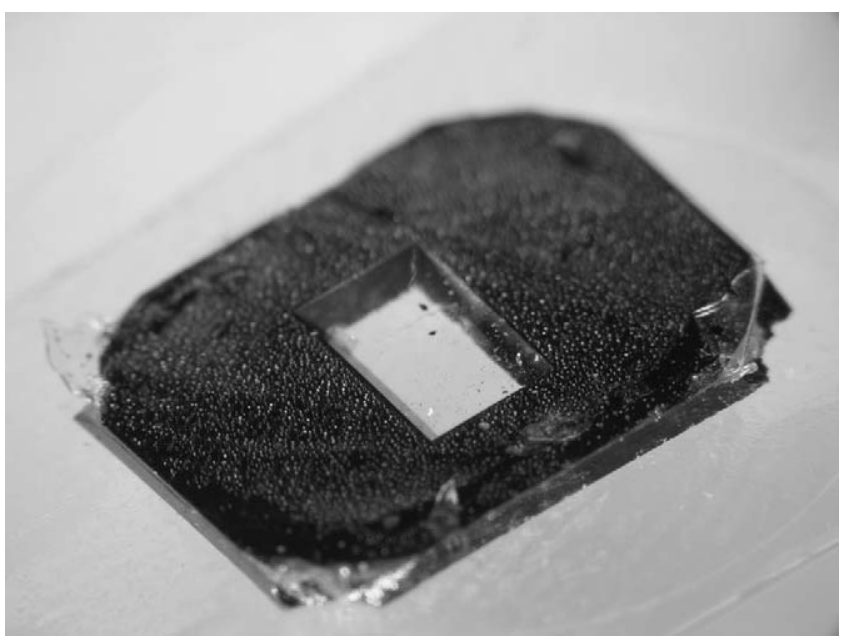

Fig. 8. The first microfabricated DEA demonstrator consisting of a 35- $\mu \mathrm{m}$ PDMS membrane bonded on a Si chip with a $\mathrm{KOH}$ wet etched rectangular cavity. The Si chip measures $9 \mathrm{~mm} \times 6.5 \mathrm{~mm}$ and has an orifice of $1.5 \mathrm{~mm} \times 2 \mathrm{~mm}$.

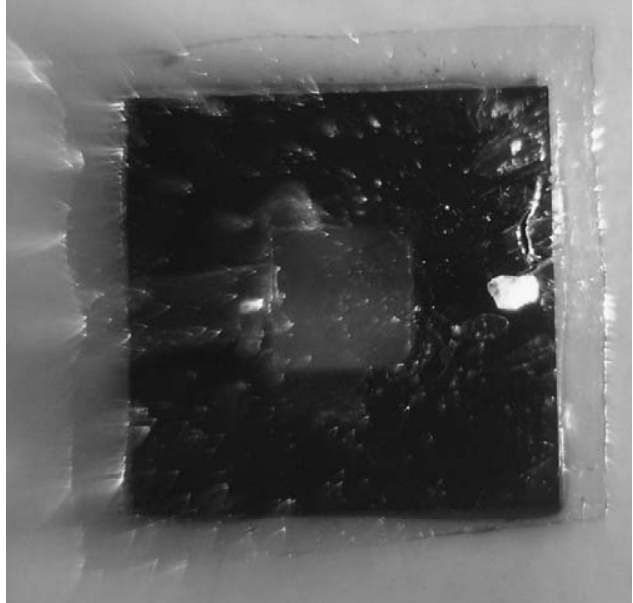

Fig. 9. The second microfabricated DEA demonstrator consisting of a $30-\mu \mathrm{m}$ PDMS membrane glued onto a Si chip with a DRIE etched square cavity. The Si chip measures $2.5 \mathrm{~mm} \times 2.5 \mathrm{~mm}$ and has an orifice of $850 \mu \mathrm{m} \times 850 \mu \mathrm{m}$.

Since the implanter used here can only implant ions into a area of about $1 \mathrm{~cm}^{2}$, we used a chip-scale process. However, wafer scale processing tests are currently ongoing using an implanter with an 8 in. diameter beam according to the following process (Fig. 10). This process purposefully results in an asymmetrical stack of layers that favors upward vertical displacements. First a sacrificial photoresist (AZ-1518) is spin coated and baked on a transfer silicon wafer (Fig. 10a). For wafer scale processing, we used this photoresist instead of PAA soluble in water to allow dicing with a saw that is sprayed with water. Then a layer of PDMS is spin coated and cured. In parallel, a silicon wafer is patterned in order to create through hole with a DRIE micromachining process. The PDMS layer is then bonded with an $\mathrm{O}_{2}$ plasma onto this patterned silicon wafer and the sacrificial photoresist layer is removed in acetone (Fig. 10b). The first metallic ions implantation is carried out on the whole PDMS surface in order to make it conducting (Fig. 10c). Then a second layer of PDMS is spin coated and cured. A lithography step is made on top to create a photoresist mask (Fig. 10d). Finally this second layer of PDMS is ion implanted and the photoresist is removed in acetone (Fig. 10e).

\section{Testing and characterization}

Implanting Ti ions into the PDMS at the conditions described above significantly lowered its surface resistivity from a starting value of more than $30 \mathrm{M} \Omega / \square$ to less than $100 \mathrm{k} \Omega / \square$. For resistivity measurements, we used two probe spring contact pins spaced by $0.5 \mathrm{~cm}$. The measurement was dependant on the pressure applied and the location of the probes. The small implanted area, about $1 \mathrm{~cm}^{2}$, was not homogeneous and had higher doses in the center of the implanted area. An investigation of the Ti implanted elastomer showed that the surface roughness was modified. Roughness measurement by AFM showed an increase in RMS roughness from 30 to $110 \mathrm{~nm}$ for the non-irradiated and irradiated elastomer, respectively.

Electrical contacts between the electrical wires and the surface of the PDMS was made with conductive adhesive (Leit- 
(a)
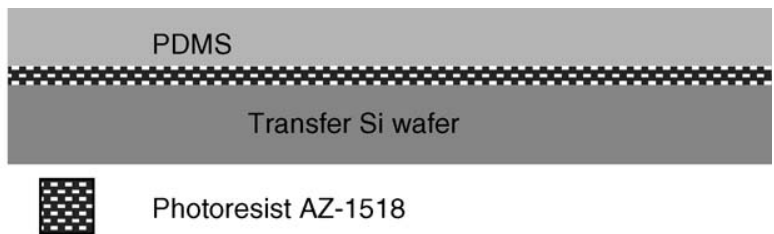

(b)

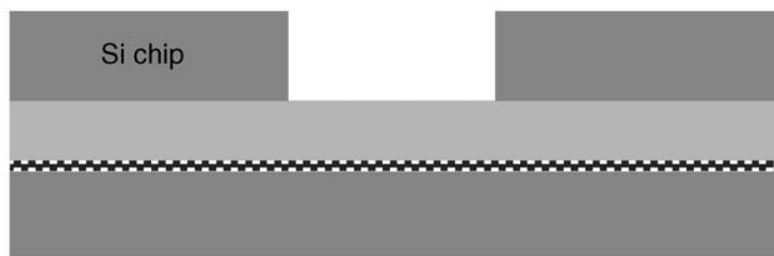

(c)

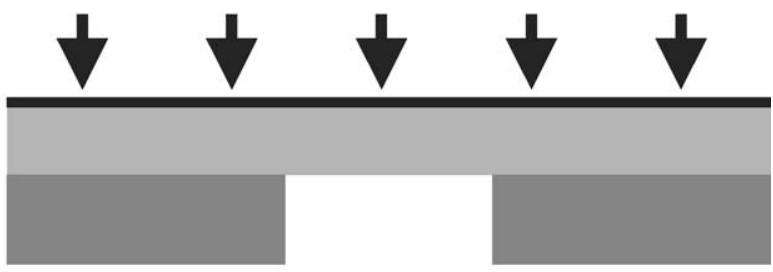

(d)

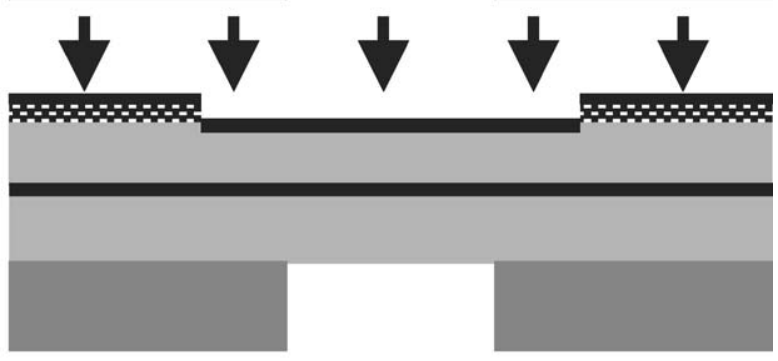

(e)

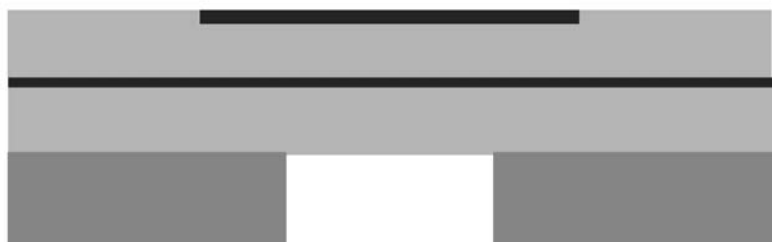

Fig. 10. Proposed wafer scale process flow for the fabrication of asymmetrical ion implanted PDMS diaphragm DEAP actuators.

C-Plast, Neubauer Chemikalien). The surface of the PDMS in contact with the silicon was electrically contacted either through the silicon chip for bonded membranes or on the parts of the membrane protruding from the silicon chip.

For testing the first generation of chips having orifices measuring $1.5 \mathrm{~mm} \times 2 \mathrm{~mm}$ (Figs. 8 and 9), the actuation voltage was increased up to the dielectric breakdown voltage of about $1.5 \mathrm{kV}$ (membrane 35- $\mu \mathrm{m}$-thick). The membrane motion was recorded on video. The complex buckling mode observed is attributed to boundary conditions asymmetries, due in part to the contacting adhesive that immobilized the membrane in one area. The maximum displacement estimated on the video was above $30 \mu \mathrm{m}$.

Above $1.4 \mathrm{kV}$, we observed large local deformations of the PDMS membrane. This behavior is similar to the pull-in effect in electrostatic MEMS [6]. Collapses are reported to occur for contractions above $30 \%$ for non-pre-stretched elastomers [6]. Localized thinning of the membrane is likely to induce dielectric breakdown and result in the failure of DEAP membrane.

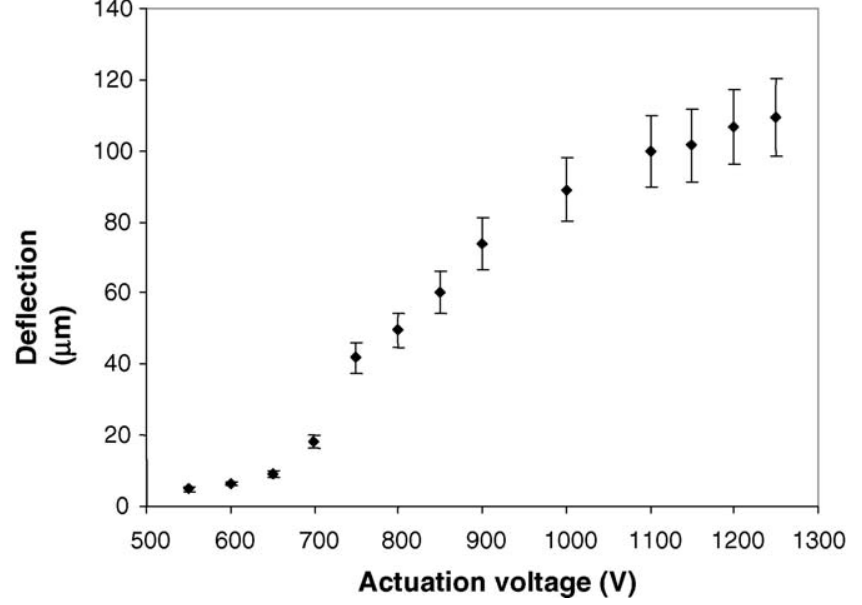

Fig. 11. Measured out-of-plane displacements vs. actuation voltage of a DEAP consisting of a Ti-ion implanted PDMS membrane bonded on a square orifice measuring $0.72 \mathrm{~mm}^{2}$. A maximum displacement of $13 \%$ of the membrane length is observed for this microfabricated device, which is comparable to performance of mature macroscale devices, showing that the implanted electrodes did not significantly increase the rigidity of the PDMS.

Therefore we estimate of the breakdown field strength of the PDMS (SmoothOn, DragonSkin) is $40 \mathrm{~V} / \mu \mathrm{m}$. Typically, the PDMS field strengths provided by manufacturers are much lower than the experimental values reported for those material shaped into membranes thinner than $100-\mu \mathrm{m}$ (over $100 \mathrm{~V} / \mu \mathrm{m}[2,14])$.

Actuators having square openings $\left(0.72 \mathrm{~mm}^{2}\right)$ were also fabricated as well and tested. These square membranes showed a buckling mode with only one maximum in the center of the membrane (i.e., pure first mode). In this case the boundary conditions were not influenced by the contacting adhesive.

The membrane displacement of the second type of chips having square openings was measured with a laser profilometer (UBM Messtechnik GMBH, Fig. 11). A step actuation from $0 \mathrm{~V}$ to the actuation voltage was made at each measurement point. We observed a maximum displacement of $110 \mu \mathrm{m}$, which represents $13 \%$ of the width of the square membrane. This percentage displacement is comparable to the best values reported for macro size diaphragm actuators [6]. Electrical breakdown occurred at about $1.3 \mathrm{kV}$, which corresponds also to an electrical field of about $40 \mathrm{~V} / \mu \mathrm{m}$ (for a $30 \mu \mathrm{m}$ thick membrane). The experiments were repeated several times and an increase of the deviation was observed at high actuation voltage. This was due to slow response time that made the maximum displacement measurement difficult. We observed response time of more than $1 \mathrm{~s}$. The electrical time constant was much lower, so the overlap response time is likely to be due to relaxation phenomenon occurring in the dielectric material.

The measured actuation voltage to create a membrane displacement of $110 \mu \mathrm{m}$ is about three times larger than predicted by FEM simulation. This discrepancy is likely due to boundary conditions and material properties that are not fully characterized for the material and fabrication process we used. Measuring the material properties of both implanted and non-implanted test samples will allow us to directly determine the amount of stiffening that ion implantation produces. However, it is clear that the 
amount of stiffening produced by ion-implantation in the actuators presented here is very limited since a ratio of displacement similar to the one of micro-devices was achieved.

\section{Conclusion}

To the best knowledge of the authors, this is the first time that micromachined metallic ion implanted dielectric electroactive polymer diaphragm (DEAP) actuators were fabricated and tested. We measured a ratio of out-of-plane displacement to diaphragm size of $13 \%$, which is approximately eight times larger than the ratio reported for a microfabricated DEAP having metal evaporated electrodes [3], where the performance was limited by the stiffness of the metal electrodes. The measured ratio is comparable to the one reported for macro size diaphragm DEAPs [6]. These observations show that ion implantation can have a very limited impact on the stiffness of the DEAP membrane. Testing revealed that the membrane modes of deformation are highly linked to the boundary conditions of the membrane.

Geometrically non-linear FEM simulations allowed computing the shape of an actuated membrane. Such simulations are highly dependant on the material properties and the boundary conditions. To be able to simulate accurately such device, the material properties must be measured on test samples having geometries close to the real device, i.e. similar membrane thickness and shape.

The tested actuators were fabricated with a chip level process. A wafer scale microfabrication process is also presented. Fabrication on the wafer level will enable individually addressing of a large number of DEAPs on a single membrane.

\section{References}

[1] S. Ashley, Artificial muscles, Sci. Am. 289 (2003) 52-59.

[2] R. Pelrine, R. Kornbluh, J. Joseph, R. Heydt, Q. Pei, S. Chiba, High field deformation of elastomeric dielectrics for actuators, Mater. Sci. Eng. C 11 (2000) 89-100.

[3] A. Pimpin, Y. Suzuki, N. Kasagi, Micro electrostrictive actuator with metal compliant electrodes for flow control applications, in: MEMS 04 Maastricht, The Netherlands, 25-29 January, 2004, pp. 478-481.

[4] S.P. Lacour, H. Prahlad, R. Pelrine, S. Wagner, Mechatronic system of dielectric elastomer actuators addressed by thin film photoconductors on plastic, Sens. Actuators A: Phys. 111 (2004) 288-292.

[5] P. Sommer-Larsen, G. Kofod, M.H. Shridhar, M. Benslimane, P. Gravesen, Performance of dielectric elastomer actuators and materials, EAPAD, SPIE, San Diego, USA, 2002, pp. 158-166.

[6] R.E. Pelrine, R.D. Kornbluh, J.P. Joseph, Electrostriction of polymer dielectrics with compliant electrodes as a means of actuation, Sens. Actuators A: Phys. 64 (1998) 77-85.

[7] Y. Wu, T. Zhang, H. Zhang, X. Zhang, Z. Deng, G. Zhou, Electrical properties of polymer modified by metal ion implantation, Nucl. Instrum. Meth. Phys. Res. Sect. B: Beam Interact. Mater. Atoms 169 (2000) 89-93.

[8] H.M. Abdel-Hamid, R.M. Radwan, A.H. Ashour, Ion beam induced changes in electrical resistivity of polymer films: the case of unplasticized poly (vinyl chloride), J. Phys. D: Appl. Phys. 35 (2002) $1183-1187$.

[9] J.H. Ha, Y.S. Cho, J.H. Lee, S.J. Lim, Polymers Surface Resistivity Improvement Using by Ion, EPAC, Vienna, Austria, 2000, pp. 2577-2579.
[10] H. Lim, Y. Lee, K.J. Kim, Reduction in surface resistivity of polymers by plasma source ion implantation, Surf. Coat. Technol. 160 (2002) $158-164$.

[11] K. Dworecki, T. Hasewaga, K. Sudlitz, S. Wasik, Modification of electrical properties of polymer membranes by ion implantation, Nucl. Instrum. Meth. Phys. Res. B 166-167 (2000) 646-649.

[12] T.W.H. Oates, D.R. McKenzie, M.M.M. Bilek, Plasma immersion implantation using polymeric substrates with a sacrificial conductive surface layer, Surf. Coat. Technol. 156 (2002) 332-337.

[13] J. Zhang, Q. Wu, X. Yu, P. Zha, H. Li, J. Zhang, Aging Stability of the morphology and wettability of titanium-implanted polytetrafluoroethylene, Phys. Stat. Solidi (A) Appl. Res. 186 (2001) 47-55.

[14] M. Benslimane, P. Gravesen, P. Sommer-Larsen, Mechanical Properties of Dielectric Elastomer Actuators with Smart Metallic Compliant Electrodes, EAPAD, SPIE, San Diego, USA, 2002, pp. 150-157.

[15] V. Linder, D.G. Byron, D. Ryan, G.M. Whitesides, Water-soluble sacrificial layers for surface micromachining, Small 1 (2005) 730-736.

\section{Biographies}

Philippe Dubois graduated in electrical engineering from the Neuchâtel University of applied science in 1991, and he received in 1998 a diploma of electronics/physics from the University of Neuchâtel. In 2003 he obtained his $\mathrm{PhD}$ on micromachined active valves and tribological studies in the group of professor de Rooij at the IMT, University of Neuchâtel. He is finishing a post-doctoral work focused on liquid valves and directional acceleration sensors, and leads projects in the group of professor de Rooij as part time researcher. Presently he leads researches on polymer actuators in the group of professor Shea in the field of microsystems for space at the EPFL.

Samuel Rosset was born in 1980 in Le Locle, Switzerland. He studied microengineering at the Swiss Federal Institute of Technology Lausanne and received his MSc degree in 2004. He is now a $\mathrm{PhD}$ student in the group of professor Shea at the EPFL working on miniaturized electroactive polymer actuators.

Sander Koster studied analytical chemistry at the University of Amsterdam and obtained a $\mathrm{PhD}$ in Fourier transform mass spectrometry at the Institute of Atomic and Molecular Physics in Amsterdam under the supervision of professor Ron Heeren. After his PhD he worked for 2.5 years at the Institute of Microtechnology in Neuchâtel, Switzerland in the group of professor de Rooij where he developed a microfluidic tool for the cultivation and chemical stimulation of living cells. At present, he is working at the University of Groningen in the Pharmaceutical Analysis Department with professor Sabeth Verpoorte as an assistant professor, where one of his activities will deal with the coupling of chips to ESI MS

Johann Stauffer graduated in micromechanical engineering from the Neuchâtel University of applied science in 2003. He is currently working at the Ion Beam Analysis Center (CAFI) in Le Locle $(\mathrm{CH})$ in the field of surface treatment and analysis.

Serguei N. Mikhaïlov obtained a PhD in physics from the Academy of Sciences in St. Petersbourg in May 1998. He is a professor at the University of Applied Sciences of Western Switzerland and the director of the Ion Beam Analyses Center (CAFI) in Le Locle. His current field of interest is ion beam analysis and surface modifications with application in micro and nanotechnology.

Massoud Dadras received his DEA from Institute of Nuclear Science and Technology, CEA, France in 1987 and his PhD from University of Orsay, France in 1990. He joined University of Neuchâtel in 1991. He is Manager of Service for Microscopy and Nanoscopy. His research activities oriented to the microstructural study and improvement of properties of thin layers used in microfabricated sensors, actuators and microsystems.

Nico-F. de Rooij joined the Institute of Microtechnology of the University of Neuchâtel, Switzerland (IMT UNI-NE), as professor and head of the Sensors, Actuators and Microsystems Laboratory in 1982. Since October 1990 till 
October 1996, he was acting as director of the IMT UNI-NE. He lectured at the Swiss Federal Institute of Technology, Zurich (ETHZ), and since 1989, he has been a part-time professor at the Swiss Federal Institute of Technology, Lausanne (EPFL). His research activities include microfabricated sensors, actuators, and microsystems. He was a member of the steering committee of the International Conference on Solid-state Sensors and Actuators and of Eurosensors. He acted as European Program Chairman of Transducers '87 and General Chairman of Transducers ' 89 . He is a member of the editorial boards for the journals Sensors and Actuators, Sensors and Materials and the IEEE Journal of Microelectromechanical Systems.
Herbert Shea has a PhD (1997) and a MA (1993) in physics from Harvard University, and a BSc (1991) in physics from McGill University. After 2 years as a post-doctoral fellow at IBM's T.J. Watson Research Center he joined Lucent Technologies' Bell Labs in Murray Hill, NJ, USA, first as a member of technical staff (1999-2001), then (2001-2004) as the technical manager of the Microsystems Technology group. Since April 2004, he is an assistant professor at the EPFL in Lausanne, Switzerland, with a focus on ultra-reliable MEMS for space applications. Research interests include nanosatellites, polymer MEMS, and the reliability and accelerated testing of silicon and polymer based microsystems. 